\title{
Performance Evaluation of Differential Global Navigation Satellite System with RTK Corrections
}

\author{
Gagandeep Purohit ${ }^{1}$, Moiz Chasmai ${ }^{2}$, Arun Barde $^{3}$, Ansari AN $^{3}$ \\ ${ }^{1}$ Research \& Development Establishment (E), India, \\ ${ }^{2}$ Research \& Development Establishment (E), India, \\ ${ }^{3}$ Research \& Development Establishment (E), India
}

\begin{abstract}
Various GNSS systems are being developed by different countries; some are regional navigation systems while others cover complete globe. Different correction techniques and augmentation systems are being used to enhance the performance of Global Navigation Satellite System (GNSS) for precisely locating the points on the surface of the earth. This paper, emphasise on GNSS selection criterion for different application. This also highlights various experiments conducted for evaluating the performance of RTK enabled differential GNSS (DGNSS) based system. The observation and analysis shows that the Real Time Kinematics (RTK) enabled DGNSS system have higher accuracy and repeatability than other augmentation systems. The repeatability over the period of time was also observed.
\end{abstract}

Key Words: GPS, GLONASS, DGNSS, RTK, INS

\section{Introduction}

GNSS is the generic term for various satellite navigation systems that provide autonomous geopositioning with global coverage. The system is used for diverse applications that include navigation of ground vehicles, ships, aircraft \& spacecraft and monitoring of shifts of the Earth's tectonic plates, etc. Existing GNSS systems are Global Positioning System (GPS) developed by US and GLObal'naya NAvigatsiomaya Sputnikova Sistema (GLONASS) developed by Russia. Europe's GALILEO, China's COMPASS Navigation Satellite System (BeiDou 1 \& 2), Japan's Quasi-Zenith Satellite System (QZSS) and Indian Regional Navigational Satellite System (IRNSS) are under development $[1,2,4]$. QZSS, BeiDou 1 and IRNSS are regional navigation system while others are global navigation systems.

Basically, a GNSS receiver determines four variables, namely latitude, longitude, altitude and time. Other information like position, speed, etc. can be derived from these four variables. There are various factors (Ionosphere, Troposphere, multiple reflections in path, gravitational force from other heavy bodies near satellite, time Synchronization, receiver noise, etc.) that affects the position accuracy of GNSS signal [5].

There are a number of techniques for reducing dilution and improving data quality. The most common methods for improving data are Ground based Augmentation system (GBAS), in which data corrections are taken from accurately surveyed ground station, Differential GNSS consisting of an additional base station receiver for differential correction. In satellite based Augmentation system (SBAS) data corrections are received from satellite instead of ground based base station. Precise Point Position (PPP) corrects data by averaging method and RTK corrects data by measuring the difference in carrier phase cycles and fractions of cycles over time by tracking the carrier signals at both the receivers at the same time [3].

Different standard formats are being used for the data transfer between augmentation system \& rover GNSS receiver based on the user needs, namely, RINEX, NGS-SP3 and RTCM SC-104 [8]. NMEA [9] standard protocols are being used to communicate between the GNSS receiver and the external device for display or any other processing.

Here we are presenting an account for series of experiments conducted on DGNSS system mounted on Tatra (8X8) vehicle. The experiments were conducted for testing the performance of DGNSS system on the basis of accuracy, repeatability at RTK and at re-acquisition of RTK. The parameters are also tested over a time period. It was observed that the achieved accuracy and repeatability are acceptable for military as well as civilian applications.

\section{System Description}

There was a requirement to locate the moving vehicle along the route precisely. A system is integrated on the vehicle which is used to bury objects of various sizes ranging from $30 \mathrm{~cm}$ to $120 \mathrm{~cm}$. Apart from the location of the moving vehicle, objects buried location is also required to be logged very precisely. The accuracy of the system which locates the objects was required to be at least less than the smallest object dimensions (30 $\mathrm{cm})$ with high precision. DGNSS was hence required to achieve this accuracy. DGNSS system with multiconstellation support, dual frequency with RTK mode was mounted on the rear end of Tatra (8X8) vehicle with 
the platform dimensions of $2500 \mathrm{~mm}$ x $9470 \mathrm{~mm}$. Base station was set at a surveyed location. The base station sends the differential corrections to the rover mounted on the vehicle through radio link. The base station includes GNSS receiver, radio modem, their respective antennas and batteries as shown in Figure 1. The rover Setup consists of Rover GNSS receiver, radio modem, and their respective antennas with similar capabilities

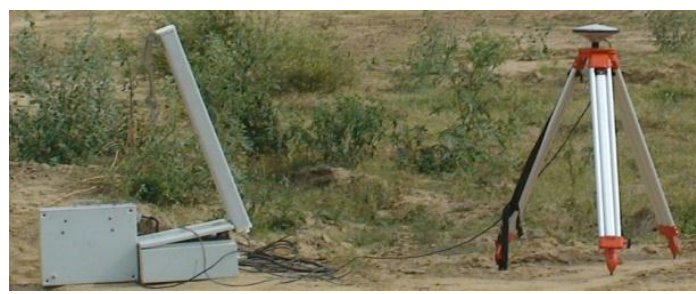

Fig. 1 Base Station Setup

\section{Performance Evaluation}

All experimentations were carried out during day time between 1130 and 1600hrs IST (GMT+05:30). The following methods are used to evaluate the performance of the system. Every method includes method description, results and analysis.

3.1 Position accuracy test for distance measurement: To evaluate the position accuracy of DGNSS system (RTK mode enabled), the base station is kept at GCP and 9 points were marked on the ground at a fixed spacing of 3 metres each (figure 2). The vehicle consisting of Rover receiver is moved along these points marked on the ground.

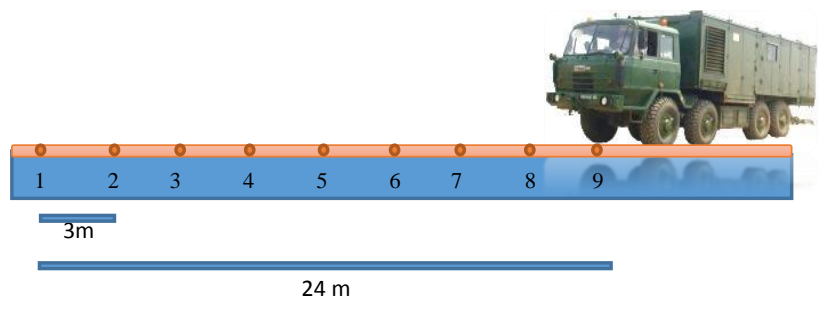

Fig. 2 Test Setup Rover Station

At each point, rover receiver observations were taken and the distances were computed by converting these latitude-longitude readings into distance (meters). The collected data and the derived distances are given in Tables $1 \& 2$ and figure 3. Here, the maximum error in the position is $0.17162 \mathrm{~m}$ for first set and $0.145121 \mathrm{~m}$ second set with average error is $0.10176 \mathrm{~m}$ for $1^{\text {st }}$ set and $0.099002 \mathrm{~m}$ for $2^{\text {nd }}$ set, which is acceptable for military ground based applications.

The major contributions in these errors are due to the manual misalignment of vehicle at the marked points on ground, the ionosphere error and receiver noise.

Table 1: Distance accuracy test readings Set 1

\begin{tabular}{|c|c|c|c|}
\hline \multicolumn{4}{|c|}{ Base Station: 18.584498101N, 73.882155539E } \\
\hline Locations & Readings & $\begin{array}{c}\text { Measured } \\
\text { Dist. }(\mathrm{m})\end{array}$ & $\begin{array}{c}\text { Actual Dist. } \\
(\mathrm{m})\end{array}$ \\
\hline 1 & $18.58186092 \mathrm{~N}, 73.87588801 \mathrm{E}$ & $\begin{array}{c}\text { Starting } \\
\text { Point }\end{array}$ & - \\
\hline 2 & $18.58186792 \mathrm{~N}, 73.87591501 \mathrm{E}$ & 3.060581 & 3 \\
\hline 3 & $18.58186459 \mathrm{~N}, 73.87594301 \mathrm{E}$ & 3.103193 & 3 \\
\hline 4 & $18.58187259 \mathrm{~N}, 73.87597001 \mathrm{E}$ & 3.094534 & 3 \\
\hline 5 & $18.58188074 \mathrm{~N}, 73.87599718 \mathrm{E}$ & 3.116795 & 3 \\
\hline 6 & $18.58188759 \mathrm{~N}, 73.87602451 \mathrm{E}$ & 3.09655 & 3 \\
\hline 7 & $18.58189292 \mathrm{~N}, 73.87605218 \mathrm{E}$ & 3.096267 & 3 \\
\hline 8 & $18.58189942 \mathrm{~N}, 73.87607951 \mathrm{E}$ & 3.087425 & 3 \\
\hline 9 & $18.58190442 \mathrm{~N}, 73.87610734 \mathrm{E}$ & 3.107568 & 3 \\
\hline
\end{tabular}

Table 2: Distance accuracy test readings Set 2

\begin{tabular}{|c|c|c|c|}
\hline \multicolumn{4}{|c|}{ Base Station: 18.584498101N, 73.882155539E } \\
\hline $\begin{array}{c}\text { Location } \\
\mathrm{s}\end{array}$ & Readings & $\begin{array}{c}\text { Measured } \\
\text { Dist. }(\mathrm{m})\end{array}$ & $\begin{array}{c}\text { Actual Dist. } \\
(\mathrm{m})\end{array}$ \\
\hline 1 & $18.58185409 \mathrm{~N}, 3.87588801 \mathrm{E}$ & $\begin{array}{c}\text { Starting } \\
\text { Point }\end{array}$ & - \\
\hline 2 & $18.58186142 \mathrm{~N}, 73.87591534 \mathrm{E}$ & 3.096105 & 3 \\
\hline
\end{tabular}




\begin{tabular}{|l|l|l|l|}
\hline 3 & $18.58186809 \mathrm{~N}, 73.87594268 \mathrm{E}$ & 3.091714 & 3 \\
\hline 4 & $18.58187442 \mathrm{~N}, 73.87597034 \mathrm{E}$ & 3.118935 & 3 \\
\hline 5 & $18.58188125 \mathrm{~N}, 73.87599768 \mathrm{E}$ & 3.096105 & 3 \\
\hline 6 & $18.58188775 \mathrm{~N}, 73.87602468 \mathrm{E}$ & 3.051801 & 3 \\
\hline 7 & $18.58189459 \mathrm{~N}, 73.87605168 \mathrm{E}$ & 3.060581 & 3 \\
\hline 8 & $18.58190125 \mathrm{~N}, 73.87607951 \mathrm{E}$ & 3.145121 & 3 \\
\hline 9 & $18.58190809 \mathrm{~N}, 73.87610718 \mathrm{E}$ & 3.131654 & 3 \\
\hline
\end{tabular}

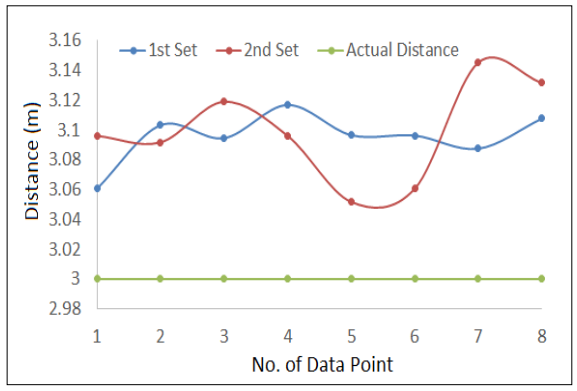

Fig. 3 Error at different point

3.2 Position repeatability test over a time period: With time, the constellation of GNSS satellites will change. Hence, the communications with previous satellites are lost and the receiver will acquire the data from new satellites. To validate the position repeatability of the receiver with time, we have to take measurements at regular intervals. Now, with the compatibility of today's receivers with both GPS and GLONASS, the receivers track more than 4 satellites at a time. This reduces the time to switch satellites.

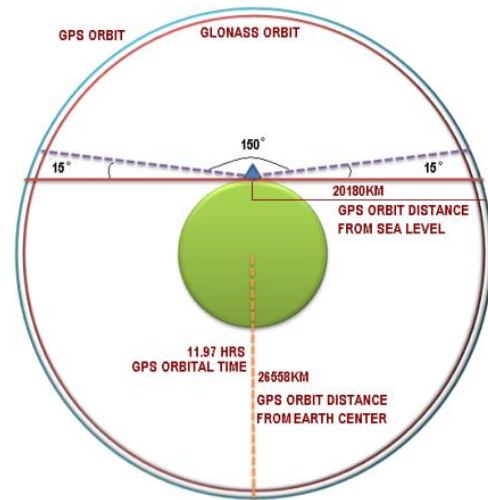

Fig. 4 Calculation of time for observations

It is required to have 150 degree $(5 \pi / 6$ radian) field of view from the observation point for receiving disturbance free signal. Therefore the length of path traversed by GPS satellite above sea level (Radius $20180 \mathrm{Km}$ ) will be $52831.11 \mathrm{~km}$ as shown in figure 4 .

The time taken by GPS satellite to cover one orbit (orbit length $166868.8 \mathrm{~km}$ ) is 11.97 hours, hence visibility of a satellite at observation point will be for 3.78 hours and thus the time for observations over time period taken as 4 hours as given in Table 3 .

It was observed that over a time period the errors are because of the data collection after switching to another set of satellites. Moreover, the errors are less in this GNSS model as the selected GNSS receiver has 50 channels, which helps in reducing the time to re-establish the link with the new set of satellites. At any given time, this receiver tracks more than 10 satellites in clear weather. This error is less compared to the ionosphere error. Manual errors are not present here as the vehicle was static for the given time period. Experiment for position accuracy over period of time was carried out for 4 hours.

Table 3: Position accuracy over time period Data

\begin{tabular}{|c|c|c|c|c|}
\hline Points & First Reading & Second Reading & $\begin{array}{c}\text { Time } \\
\text { duration }\end{array}$ & Error (m) \\
\hline A & $18.581908087 \mathrm{~N}$, & $18.581908070 \mathrm{~N}$, & $4 \mathrm{hrs}$ & 0.038 \\
& $73.876107177 \mathrm{E}$ & $73.876107506 \mathrm{E}$ & & \\
\hline B & $18.581895920 \mathrm{~N}$, & $18.581895920 \mathrm{~N}$, & $4 \mathrm{hrs}$ & 0.0295 \\
& $73.876052011 \mathrm{E}$ & $73.876052011 \mathrm{E}$ & & \\
& & & & \\
\hline
\end{tabular}


3.3 Position repeatability test after repositioning: Position accuracy repeatability has to be checked for better performance of the receiver. It also quantifies rousing error during positioning of the receiver. Following process is carried out for in house experiment in RTK mode.

Three positions A, B and C as shown in figure 5 are marked and observations were recorded. Again the receiver was kept at all these positions and the observations were taken in terms of latitude-longitude. Both the observations at each point are compared to establish the repeatability. The sample data collected is given in Table 4 and the comparison of the actual distances between the points and the distances derived is given in Table 5. With RTK corrections, the average variation calculated is $0.036961 \mathrm{~m}$ and the maximum variation calculated is $0.0511357 \mathrm{~m}$.

The major contributing factors in this error are manual misalignment of the vehicle at the marked points on the ground, the ionosphere error and receiver noise.

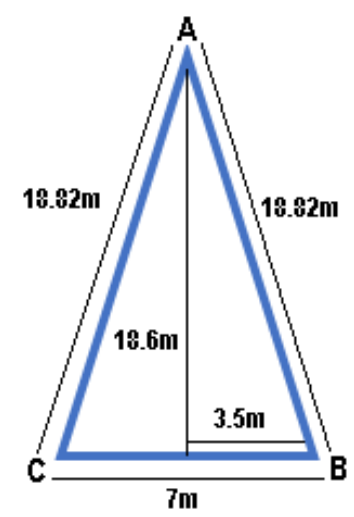

Fig. 5. Schematic diagram of Position repeatability test after repositioning

Table 4: Repositioning Data

\begin{tabular}{|c|c|r|c|}
\hline Points & First Reading & Second Reading & $\begin{array}{c}\text { Error } \\
(\mathrm{m})\end{array}$ \\
\hline A & $18.58189892 \mathrm{~N}$ & $18.58189925 \mathrm{~N}$ & 0.046 \\
& $73.87593584 \mathrm{E}$ & $73.87593568 \mathrm{E}$ & \\
\hline B & $18.58183692 \mathrm{~N}$ & $18.58183709 \mathrm{~N}$ & 0.018 \\
& $73.87595084 \mathrm{E}$ & $73.87595084 \mathrm{E}$ & \\
\hline C & $18.58190809 \mathrm{~N}$ & $18.58190809 \mathrm{~N}$ & 0 \\
& $73.87610718 \mathrm{E}$ & $73.87610718 \mathrm{E}$ & \\
\hline
\end{tabular}

Table 5: Repositioning Data in form of distances

\begin{tabular}{|c|c|c|r|}
\hline Points & $\begin{array}{c}\text { Actual } \\
\text { distance } \\
(\mathrm{m})\end{array}$ & $\begin{array}{c}\text { First set } \\
\text { Distance } \\
(\mathrm{m})\end{array}$ & $\begin{array}{c}\text { Second set } \\
\text { Distance } \\
(\mathrm{m})\end{array}$ \\
\hline $\mathrm{AB}$ & 18.82 & 18.88 & 18.87 \\
\hline $\mathrm{BC}$ & 18.82 & 18.8547665 & 18.8711357 \\
\hline $\mathrm{CA}$ & 7 & 7.00974876 & 7.03186995 \\
\hline
\end{tabular}

3.4 Position repeatability test after re-acquisition: Initially, after getting RTK fix at a given position of the vehicle, the GNSS receiver was turned OFF and restarted to get the new fix. It takes few seconds to give stable and accurate reading after initialization and reacquisition. The readings are compared to get the position repeatability.

In case of DGPS/GBAS mode, the error is comparatively higher while in case of RTK float error is moderate. RTK fix gives best repeatability after re-acquisition of fix. The observations are shown in table 6 given below.

Table 6: Position repeatability test after re-acquisition

\begin{tabular}{|c|c|c|c|c|}
\hline & Set 1 & Set 2 & $\begin{array}{c}\text { Error set1 } \\
\mathrm{m}\end{array}$ & $\begin{array}{c}\text { Error set2 } \\
\mathrm{M}\end{array}$ \\
\hline Initial value with RTK fix & $18.581848253 \mathrm{~N}$ & $18.581848253 \mathrm{~N}$ & - & - \\
& $73.875997011 \mathrm{E}$ & $73.875997177 \mathrm{E}$ & & 0.5 \\
\hline DGPS/GBAS & $18.581853637 \mathrm{~N}$ & $18.581848587 \mathrm{~N}$ & 0.6 & \\
& $73.875996344 \mathrm{E}$ & $73.875992677 \mathrm{E}$ & & 0.34 \\
\hline RTK Float & $18.581847920 \mathrm{~N}$ & $18.581851253 \mathrm{~N}$ & 0.31 & 0.06 \\
\hline RTK Fix & $73.875994177 \mathrm{E}$ & $73.875996511 \mathrm{E}$ & & 0.09 \\
\hline
\end{tabular}


As all these tests were carried out during day time, HDOP (Horizontal Dilution of Position) was more due to expansion in the width of ionosphere layer. This expansion arises from the increase in temperature. If these experiments are carried out at night then these errors would decrease by 8-10 times.

\section{Conclusion}

DGNSS is an accurate and reliable tool for precise location marking and recording system. However, the RTK aimed to improve the performance of the DGNSS systems even further. DGNSS with RTK corrections give an accuracy of sub-decimetres which meets the high end requirements of military applications. The repeatability and consistency in results during repositioning of entire vehicle and re-acquisition of RTK corrections is also under decimetre level. It is also seen that accuracy during RTK correction is much better than DGPS/GBAS and RTK float. However, manual errors while positioning the vehicle cannot be ruled out.

\section{Acknowledgement}

The authors are grateful to Dr. S Guruprasad, Director, R\&DE (E), Pune for allowing us to publish this work. We express our sincere thanks and appreciation to Mr. VV Parlikar for their constant encouragement, guidance and scientific discussions.

\section{References}

[1]. A Brief History of Global Navigation Satellite Systems, The Journal Of Navigation, The Royal Institute of Navigation, Vol 65, No. 1, Jan 2012, pp. 1

[2]. Jean Marie Zogg, GPS Basics: Introduction to the system application overview, 2002, GPS-X-02007

[3]. Real Time Kinematic Surveying: Training Guide, Trimble, Part No. 33142-40, Revision D, September 2003

[4]. http://en.wikipedia.org/wiki/Satellite_navigation

[5]. http://precisiontracking.com.au/blog/factors-affecting-gps-accuracy/

[6]. Spletter Deutsches Zentrum Für Luft-und Raumfahrt (DLR), ARAIM: Utilization of Modernized GNSS for Aircraft-Based Navigation Integrity Alexandru (Ene)

[7]. Field Techniques Manual: GIS, GPS and Remote Sensing, Chapter 6, The Global Positioning System (GPS): Principles \& Concepts

[8]. Ahmed El-Rabbany, Introduction to GPS: The Global Positioning System, Mobile Communications Series, Artech House, BostonLondon

[9]. Klaus Betke, The NMEA 0183 Protocol, August 2001 